\title{
La bioética y el Plan Nacional de Desarrollo de México
}

\author{
Isabel Sada-Ovalle $\bowtie$
}

Instituto Nacional de Enfermedades Respiratorias Ismael Cosío Villegas, Ciudad de México.

Trabajo recibido: 03-XI-2014; aceptado: 05-XI-2014

\begin{abstract}
Para educar a un pueblo se precisa actitud moral de los hombres del poder Lázaro Cárdenas
\end{abstract}

La bioética inicia en México como un movimiento histórico, multidisciplinario, en el campo de la biomedicina y las ciencias humanísticas. Con los años, se ha convertido en un campo de estudio a nivel profesional que cuenta con técnicas de entrenamiento y certificación por expertos. Ética y bioética son palabras que no significan lo mismo para todos en este país. Generalmente existe una acepción amplia que entiende a la bioética como el estudio moral sobre la vida, la muerte, la salud, el medio ambiente, etc., y una acepción más restringida que enfoca a la bioética en la ética de la medicina. La experiencia cotidiana nos ha demostrado que pueden ser muchos los malentendidos que se presentan al confundir lo ético con lo religioso, o con los usos y costumbres de una región determinada, incluso es común ver que existe confusión entre aquello que es ético y lo que es moral. La ética es una disciplina filosófica, una rama de la filosofía, que reflexiona sobre un fenómeno humano al que llamamos moral. Por ejemplo, una enseñanza moral es: «no debemos matar a un ser humano salvo que sea en legítima defensa»; ahora, cuando preguntamos ¿por qué no se debe matar o hacer daño? entramos al ámbito de la ética en el sentido de la filosofía moral. La ética como filosofía no inventa a la moral, no tiene como finalidad generar conocimiento que permita diseñar leyes o normas sociales; al contrario, aclara en qué consiste la moral, fundamenta las creencias y sus prácticas.

El Plan Nacional de Desarrollo (PND) que hizo público el Presidente Enrique Peña Nieto al inicio de su sexenio, define a la bioética como «la rama de la ética aplicada que reflexiona, delibera y hace planteamientos normativos y de políticas públicas, para regular y resolver conflictos en la vida social, especialmente en las ciencias de la vida, así como en la práctica y en la investigación médica que afecten la vida en el planeta, tanto en la actualidad como en futuras generaciones", adicionalmente cita: «La bioética es un elemento que también favorece el desarrollo de la democracia por ser un ámbito participativo e incluyente. Tiene amplio impacto social, tanto en aspectos de protección de la salud y la dignidad humana, como en las actividades de investigación en seres vivos, el cuidado del entorno y la vida en general, al igual que en los campos científicos y tecnológicos, lo cual incide en la formulación y el diseño de políticas públicas». La forma en que están redactados estos dos fragmentos nos hacen pensar que la bioética podría funcionar como un manual de procedimientos a través del cual vamos a ser capaces de diseñar muy buenas políticas públicas; sin embargo, las buenas políticas públicas en una sociedad democrática son el resultado de un proceso largo y complejo cuyo motor es la sociedad, una sociedad que por lo tanto debe de contar ya con un enfoque ético en su actuar.

El día 2 de diciembre del año 2012 se firmó el Pacto por México; este documento estipula que el gobierno tiene el compromiso de impulsar todas aquellas iniciativas y reformas que medien acciones efectivas que MEJOREN al PAÍS, esto incluye el fortalecimiento de una sociedad de derechos y libertades donde se enfatiza en el derecho a la protección de la salud y la salvaguarda de la dignidad humana, aspectos que la bioética retoma y lleva a la práctica cotidiana.

Las líneas de acción que se proponen en este pacto dicen así:

1. Consolidar la cultura bioética en México a partir del análisis laico, plural e incluyente de los dilemas bioéticos a través de la Comisión Nacional de Bioética (CONBIOÉTICA).

2. Fomentar la protección de la dignidad y los derechos de las personas en la prestación de servicios de salud y en la investigación, considerando la integridad 
científica, la equidad y la protección de grupos en situación de vulnerabilidad.

3. Promover la consolidación de las comisiones estatales de bioética, los comités hospitalarios de bioética y los comités de ética en investigación, como medios garantes de la difusión de la cultura bioética, la aplicación de los principios bioéticos en el quehacer de la salud y en las ciencias de la vida, así como en la protección de los sujetos que participan en la investigación.

4. Impulsar la observancia de criterios de bioética de nivel mundial, posicionando a México como un referente internacional en la materia.

Hace un par de días asistí al foro: Hacia la Estrategia Nacional de Capacitación y Formación en Bioética. En este foro, escuchamos las ponencias de diversos profesionistas involucrados en la implementación de las comisiones estatales de bioética, la enseñanza de la bioética a nivel de pre y posgrado, filósofos, y miembros de los comités de ética en investigación y hospitalarios de bioética de instituciones públicas y privadas.

Considero que es importante hacer énfasis en algunas situaciones que fueron ampliamente discutidas en este foro:

1. CONBIOÉTICA es una institución que tiene personal altamente preparado en el campo de la bioética, una biblioteca con un gran acervo y, sobre todo, tienen la disposición para ayudar a las personas y a las instituciones en todos los aspectos posibles.

2. Las universidades públicas y privadas (Universidad Nacional Autónoma de México, Universidad de Querétaro, Anáhuac, Panamericana, ITESM, etc.) cuentan con programas de posgrado que responden a la gran necesidad de formar gente en este campo.

3. En México como en otros países de centro y Sudamérica carecemos de bioeticistas.

4. Es un acuerdo entre los encargados de esta enseñanza que el estudio de la bioética debe de ser incluido en la síntesis curricular de todas las licenciaturas.

5. El uso de las nuevas tecnologías de información y comunicación (TICs) nos permite adquirir conocimientos en el campo de la filosofía y la bioética con gran facilidad y sin la necesidad de necesitar una gran inversión económica (en la mayor parte de los Massive Open Online Course [MOOC]).

6. Es importante educar al personal de las instituciones en derechos humanos y bioética con la finalidad de contar con los elementos que permitan poder responder a las exigencias de los tiempos actuales. A partir de esta capacitación, es que se puede de- sarrollar el hábito de introspección que implica vivir un proceso de auténtica transformación intelectual y moral que nos llevará a reconceptualizar nuestra misión como trabajadores de la salud.

7. Al ser una recomendación presente en el PND, las instituciones deberían fomentar y apoyar (en tiempo y económicamente) a los miembros de los comités de ética y bioética con la finalidad de reducir los conflictos hospitalarios y de investigación que se presentan de manera cotidiana.

México es un país plural y, por este motivo, el estudio y la enseñanza de la bioética es un proceso más complejo ya que debemos tener en cuenta el origen cultural, geográfico y las creencias de cada individuo. La suma de las experiencias vividas aunado a la memoria de tiempos pretéritos está presente en todos y cada uno de nosotros. Es así que la conciencia del mexicano se ha formado y deformado a lo largo de los años teniendo como base la suma de experiencias colectivas que en ocasiones son bastante desfavorables. A lo largo de los años, hemos observado que la educación pública es precaria y la cultura insuficiente. Es entendible, por consecuencia, que el mexicano tenga una valoración negativa de su nacionalidad; y éste, es un factor crítico que debe tomarse en cuenta al momento de educar en ética y bioética a los hombres y mujeres de México.

La investigación científica en nuestro país es un componente indispensable de la cultura y la vida social, ya que incluso aquellas comunidades que no participan activamente en la generación de conocimiento científico se pueden ver beneficiadas del mismo en algún momento de sus vidas. La comunidad científica en el campo de la medicina ha sido quizá la más interesada en el estudio y aprendizaje de la bioética y esto es probablemente consecuencia de que fueron la medicina clínica y la investigación biomédica las responsables de estudiar y reflexionar sobre la forma de tratar adecuadamente al enfermo, reconociendo su dignidad personal y comportándose de modo acorde.

El aprendizaje por competencias también aplica al campo de la bioética que se enseña a nivel de pre y posgrado. CONBIOÉTICA, el Programa Universitario de Bioética de la UNAM, el Observatorio de Bioética y Derecho de la Universidad de Barcelona, el Programa Regional de Bioética de la Organización Panamericana de la Salud (OPS), la Organización de las Naciones Unidas para la Educación, la Ciencia y la Cultura (UNESCO), el Observatorio de Bioética de la Universidad Católica de Valencia, la Universidad de Harvard, la Universidad de Oxford, la del Centro Interdisciplinario de Estudios en Bioética de la Universidad de Chile, los Institutos Nacionales de Salud (NIH) y el Instituto Tecnológico de 
Massachusetts (MIT), son algunas de las instituciones que brindan diversos recursos en línea que podemos utilizar para formarnos en el campo de la bioética.

Para todos los que hacemos algún tipo de investigación biomédica, epidemiológica o clínica es importante la reflexión en ética y publicaciones científicas. Hasta hace algunos años considerábamos que los científicos procedían de modo cabal y honesto, sin plagio, sin repetir lo ya publicado y sin adulterar los datos. La distribución de mérito por autoría se ha convertido en tema de debate, especialmente en los grandes trabajos multicéntricos que requieren de un gran número de participantes. Aquellos estudios que involucran sujetos humanos han incurrido en la transgresión de normas elementales de protección de los individuos o de su dignidad. Algunos ensayos clínicos aleatorios y multicéntricos e internacionales que reciben financiamiento de la industria farmacéutica han modificado el fin último de la medicina, aliviar el sufrimiento del paciente, lo han cambiado por el poder económico y prestigioso de las empresas financiadoras. El sesgo que observamos en las publicaciones que derivan de la industria farmacéutica, puede ocultar o no informar los hallazgos negativos o las complicaciones de los nuevos tratamientos. Los incentivos que reciben las instituciones, los investigadores y los médicos, aunado a la necesidad económica de los participantes (en su gran mayoría provenientes de sitios con muy escasos recursos) es la vía que facilita el quehacer de este tipo de investigación poco ética.

Es así que la enseñanza y el estudio de la bioética en México son algunos de los desafíos más importantes que enfrentamos en la actualidad. Ante esta gran necesidad de formadores, han surgido en los últimos años ofertas de cursos, talleres y seminarios; incluso programas de posgrado que son impartidos por individuos que se consideran a sí mismos como expertos en bioética cuando no cuentan con un entrenamiento avalado con certificados que tenga el respaldo de una institución oficial y de prestigio. Es indispensable contar con programas que utilicen competencias bioéticas entendidas como las capacidades y actitudes que posibiliten el diálogo entre profesionales, entre profesionales y usuarios y entre usuarios y políticos. La mayor parte de las competencias en bioética tienen que ver con la honestidad, la solidaridad, la actitud de beneficencia, el altruismo; que a su vez, son parte de la conducta ética de cada profesionista.

La bioética en México debe ser un puente entre las personas (abogados, maestros, médicos, deportistas, etc.), entre grupos políticos, entre científicos, entre los diferentes grupos indígenas, entre los intereses sociales.

Incluir a la bioética no como una herramienta de trabajo o como un método facilitador de las relaciones interpersonales y laborales; sino como un estilo de vida, es algo que debe alentar la iniciativa de crecimiento de este país tan lastimado por los hechos actuales, tan deprimido y desesperanzado en su juventud, tan lleno de potencial para desarrollarse.

La bioética nos permite usar las deliberaciones de carácter moral para formular, articular y fundamentar principios y posturas en nuestra vida personal y laboral. ¡Implementémosla!

Finalmente, los siguientes links que aparecen son para aquellos lectores interesados en leer con respecto a este fascinante tema de la bioética:

1. www.actabioethica.cl

2. www.paho.org/bioetica

3. www.libros.uchile.cl

4. www.unesdoc.unesco.org

5. http://www.bioetica.unam.mx/index.html

6. http://www.bioeticayderecho.ub.edu/

7. http://colegiodebioetica.org.mx/wp/

8. www.who.int

9. www.bioeticachile.cl

\section{$\triangle$ Correspondencia:}

Dra. Isabel Sada-Ovalle, Laboratorio de Inmunología Integrativa, Instituto Nacional de Enfermedades Respiratorias Ismael Cosío Villegas. Calzada de Tlalpan Núm. 4502, Colonia Sección XVI. México, D.F., 14080.

Correo electrónico: isadamx@iner.gob.mx; isadamx@gmail.com

El autor declara no tener conflicto de interés. 